\title{
Morphology of distal radius curvatures: a CT-based study on the Malaysian Malay population
}

\author{
Taran Singh Pall Singh ${ }^{1}$, MMed, Abdul Nawfar $\underline{\text { Sadagatullah }}^{1}$, MMed, Abdul Halim $\underline{\text { Yusof }}{ }^{1}$, MMed
}

\begin{abstract}
INTRODUCTION The purpose of this study was to examine the differing curves of the volar distal radius of healthy Malaysian Malays, so as to obtain detailed morphological information that will further the understanding of volar plate osteosynthesis in Malaysian Malays.

METHODS Computed tomography with three-dimensional reconstruction was performed on the wrists of 16 healthy Malaysian Malay volunteers. Profile measurements were made using a software program. A novel parameter, the pronator quadratus curve angle, was explored and introduced in this study. Interclass correlation coefficients were calculated to assess the level of agreement between the data collected by the principal investigator and that collected by an independent radiologist.

RESULTS The mean \pm standard deviation of the arc radii on the radial aspect was $17.50^{\circ} \pm 5.40^{\circ}$, while the median (interquartile range [IQR]) of the arc radii on the ulnar aspect was $25.27^{\circ}\left(\right.$ IQR $\left.5.80^{\circ}\right)$. The mean \pm standard deviation of the curvature of the pronator quadratus line was $40.52^{\circ} \pm 2.48^{\circ}$. The arc radii on the radial aspect was significantly lower than the arc radii on the ulnar aspect $(p=0.001)$. Different radial and ulnar arcs were observed in $56.25 \%$ of the radii; the arc was deeper on the ulnar aspect in $93.75 \%$ of the radii.

CONCLUSION Based on the findings of this study, the likelihood of achieving anatomical reduction with uniformly curved, fixed-angle volar plates is questionable. Changes in the design of these implants may be needed to optimise their usage in the Malaysian Malay population.
\end{abstract}

Keywords: arc radii, distal radius fracture, distal radius morphology, volar plating

\section{INTRODUCTION}

Approximately $30 \%$ of all patients with distal radius fractures, including those treated operatively with volar plate osteosynthesis, heal with unsatisfactory functional and anatomic outcomes. ${ }^{(1)}$ Complications of volar plate osteosynthesis have been well enumerated in the literature. ${ }^{(1-10)}$ However, only recently were variations in the morphology of the distal radius observed. ${ }^{(11,12)}$ Such variations may, directly or indirectly, influence the outcome of osteosynthesis for distal radius fractures that uses the current generation of uniformly prebent, fixed-angle volar plates. Hence, it is essential to gather information on these morphological variations in the distal radius to broaden our understanding of volar plate osteosynthesis.

The main objectives of the present study were: (a) to define the curvature of the arc radii on the radial and ulnar aspects of the distal radius in a Malaysian Malay population; and (b) to identify any significant differences between the aforementioned curvatures. In addition, the present study also aimed to define the curvature of the pronator quadratus (PQ) line, which is the boundary between the PQ muscle and the proximal extent of the volar fibrous tissue of the wrist joint. This line can be considered as the distal safe limit for placement of a volar plate. ${ }^{(13)}$ The greatest width of the distal radius in the coronal plane and the width of the distal radius $4 \mathrm{~cm}$ proximal to the tip of the radial styloid process were also measured in the present study. We conducted this explorative study on individuals of Malay ethnicity, as Malays are the predominant race in Kelantan, Malaysia, and more than
$90 \%$ of the patients with distal radius fractures in our institution were of Malay ethnicity.

\section{METHODS}

In this cross-sectional study, the first 16 volunteers who fulfilled the inclusion criteria and provided informed, written consent were selected. Volunteers must be of Malay ethnicity and have fused distal radius growth plates to be included in the present study. Volunteers with the following characteristics were excluded: (a) metabolic bone disease; (b) history of degenerative, inflammatory or infective diseases around the wrist; (c) history of fracture or dislocation around the wrist; (d) bone tumours around the wrist; and (e) radiological evidence of abnormality or deformity of the distal radius. Plain radiography (anteroposterior and lateral views) was done on the right wrist for all volunteers to check for these characteristics. Volunteers who were pregnant were also excluded due to the risk of radiation exposure.

As the present study involved the use of computed tomography (CT) on healthy volunteers, ethical approval was obtained from the Ethics Committee of Universiti Sains Malaysia, Kubang Kerian, Kelantan, Malaysia. Noncontrast CT was performed on the right wrist of all volunteers. SOMATOM Definition AS+ in 128-slice configuration (Siemens AG, Wittelsbacherplatz, Muenchen, Germany) was used in the present study. All scans were performed by the same experienced radiographer, using standardised imaging protocol (pitch 0.85; scanning parameters $120 \mathrm{kV}$, $112 \mathrm{mAs}$ ). Three-dimensional (3D) reconstruction was done using

${ }^{1}$ Department of Orthopaedics, Universiti Sains Malaysia, Kelantan, Malaysia

Correspondence: Dr Taran Singh Pall Singh, Lecturer \& Orthopaedic Surgeon, Department of Orthopaedics, Universiti Sains Malaysia, 16150, Kubang Kerian, Kelantan, Malaysia.taran@usm.my 
postprocessing in Syngo 3D (Siemens AG, Wittelsbacherplatz, Muenchen, Germany) and the bones were viewed using a volume rendering technique. The images were reconstructed from a 0.6-mm slice thickness on soft tissue window (i.e. Kernel U30u). Digital subtraction of the ulna and carpal bones was done.

Profile measurements of the distal radius were made using the 3D-reconstructed CT images and the OsiriX imaging software, an open source DICOM viewer (Pixmeo, Geneva, Switzerland). Each parameter was measured three times and the mean measurement was used as the definite measurement. The 'length' tool was used for width measurements and the 'dynamic angle' tool was used for the angle measurements. The readings were taken after the tools were calibrated, from pixels to centimetres, using the sagittal CT image. The following measurements were taken on all 3D-reconstructed distal radius images: (a) arc radii on the radial aspect (dynamic angle in degrees) - one point was made at the volar rim, one point along the curve $2 \mathrm{~cm}$ from the volar rim and two points along the curve (Fig. 1); (b) arc radii on the ulnar aspect (dynamic angle in degrees) - one point was made at the volar rim, one point along the curve $2 \mathrm{~cm}$ from the volar rim, and two points along the curve (Fig. 2); (c) curvature of the PQ line (dynamic angle in degrees) - one point was made at

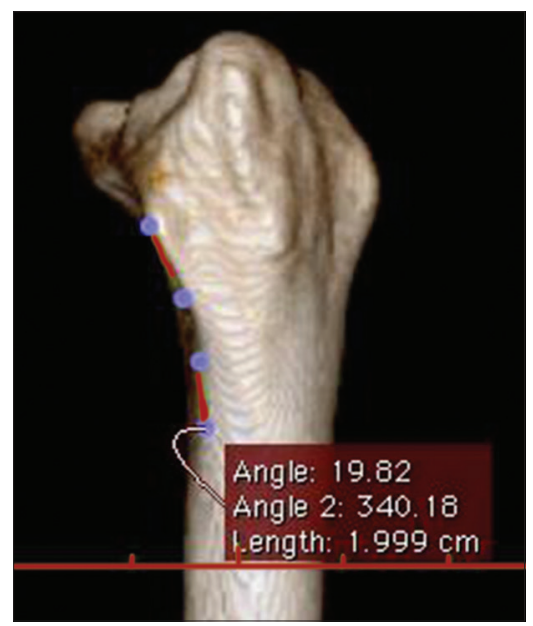

Fig. 1 3D-reconstructed CT image shows arc radii measurement on the radial aspect of a distal radius.

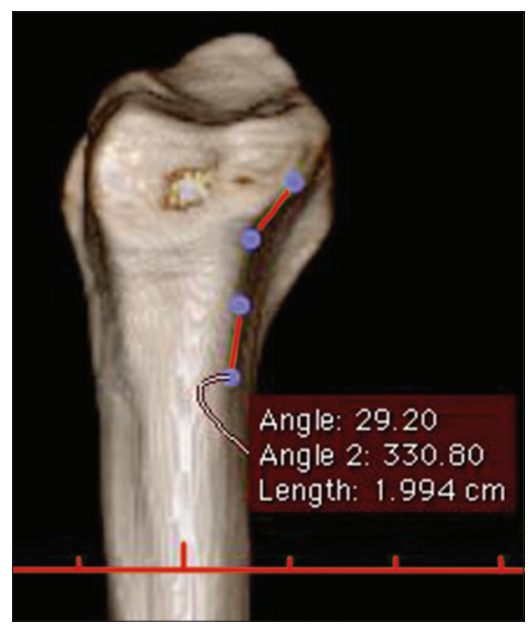

Fig. 2 3D-reconstructed CT image shows arc radii measurement on the ulnar aspect of a distal radius. the volar radial tuberosity, one point where the ulna was most prominent at the base of the volar lunate facet, and two points along the curved ridge (Fig. 3); (d) greatest width of the distal radius (in centimetres) - the length across two points that marked the greatest width of the distal radius (Fig. 4); and (e) width of the distal radius $4 \mathrm{~cm}$ proximal to the tip of the radial styloid process (in centimetres) - a $4 \mathrm{~cm}$ length from the tip of the radial styloid was measured, then the width of the radial shaft was measured across the two points that marked the width at that level (Fig. 5).

All measurements were made and collected by the principal investigator. To validate the data collected, a complete and independent set of measurements was made by a radiologist who subspecialises in musculoskeletal radiology. The interclass correlation coefficients were calculated to assess the agreement between the measurements collected by the principal investigator and those collected by the radiologist. Continuous variables were presented as mean \pm standard deviation, while categorical variables were presented as frequency (percentage). Descriptive statistics were used to define (a) the curvatures of the arc radii on the radial and ulnar aspects; (b) the curvatures of the PQ line; and (c) width measurements. Wilcoxon signed-rank test was used for paired samples (i.e. to identify the difference between the arc radii

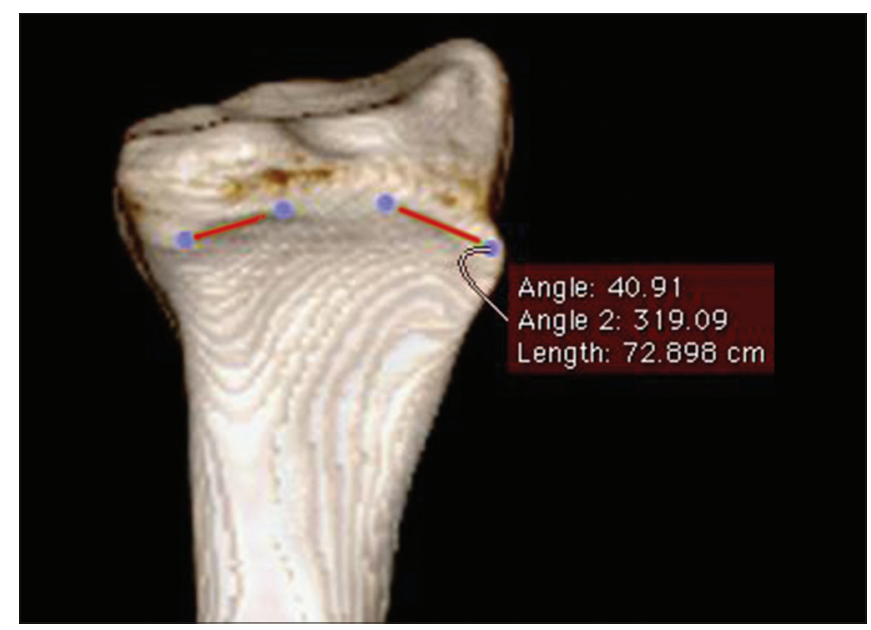

Fig. 3 3D-reconstructed CT image shows measurement of the curvature of the pronator quadratus line.

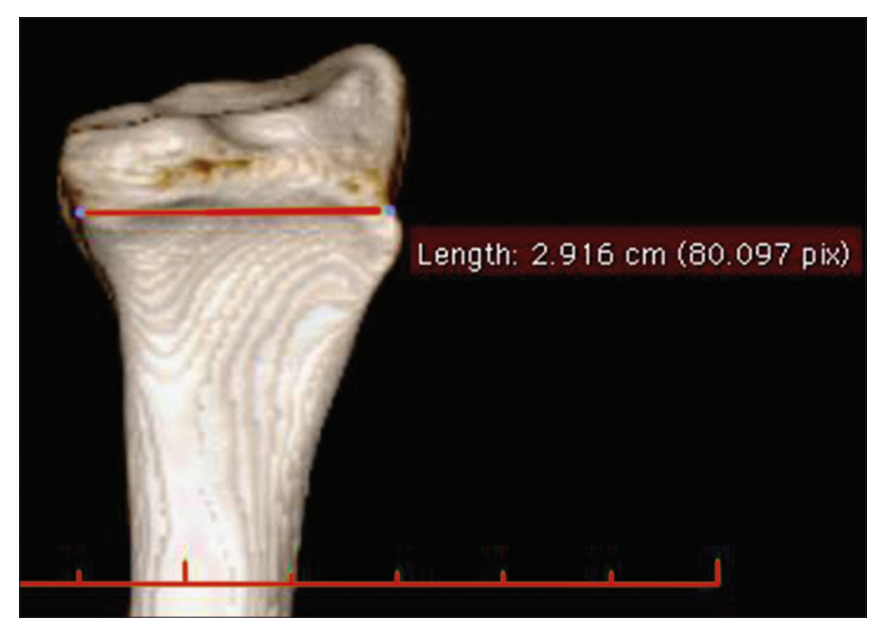

Fig. 4 3D-reconstructed CT image shows measurement of the greatest width of the distal radius. 


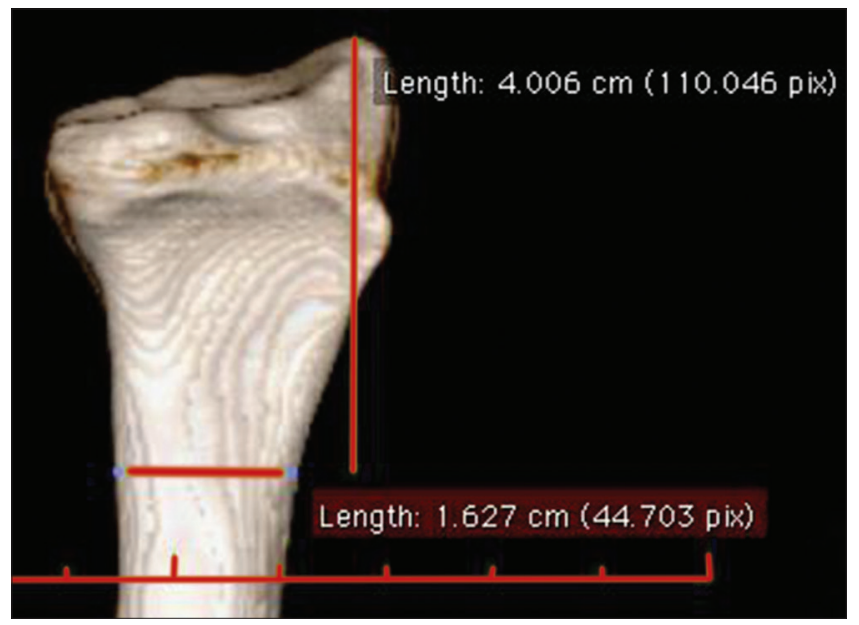

Fig. 5 3D-reconstructed CT image shows measurement of the width of the distal radius $4 \mathrm{~cm}$ proximal to the tip of the radial styloid process.

on the radial and ulnar aspects). Mann-Whitney $U$ test was used for independent samples (i.e. to identify the difference between the measurements of the PQ line in males and females) and to identify the difference between measurements in relation to handedness. All remaining analyses were done using an independent sample $t$-test. The significance level was set at $p$-value $<0.05$.

\section{RESULTS}

In the present study, the interclass correlation agreement was high between all the measurements made by the researcher and the radiologist, except for the curvature of the PQ line, which only had moderate interclass correlation agreement (Table I). The age distribution of the volunteers was skewed, with a median (interquartile range [IQR]) age of 23.00 (IQR 3.00) years. Among the 16 volunteers, nine were female and seven were male; 14 were right-hand dominant. The mean \pm standard deviation of the arc radii on the radial aspect was $17.50^{\circ} \pm 5.40^{\circ}$ (range $\left.9.22^{\circ}-29.00^{\circ}\right)$. The median of the arc radii on the ulnar aspect was $25.27^{\circ}\left(\mathrm{IQR} 5.80^{\circ}\right.$; range $\left.16.56^{\circ}-41.27^{\circ}\right)$. The mean \pm standard deviation of the curvature of the PQ line was $40.52^{\circ} \pm 2.48^{\circ}$ (range $34.48^{\circ}-45.75^{\circ}$ ). The mean of the greatest width of the distal radius was 2.80 (range $2.26-3.21$ ) $\mathrm{cm}$. The mean width of the distal radius $4 \mathrm{~cm}$ proximal to the radial styloid process tip was 1.60 (range $1.08-1.92$ ) $\mathrm{cm}$. The arc radii on the radial aspect was found to be significantly lower than the arc radii on the ulnar aspect $(Z=-3.464, p=0.001)$. If we define 'different arc angle' as a difference of $5^{\circ}$ or more, only seven radii would have had the same arc angle on both the radial and ulnar aspects (the remaining nine radii would have had different arc angles). The arc angle was wider on the ulnar aspect in fifteen radii.

No significant difference was observed between the volunteers who were male and those who were female in terms of the arc radii on the radial aspect $(p=0.579)$, arc radii on the ulnar aspect $(p=0.262)$, curvature of the PQ line $(p=0.791)$, greatest width of the distal radius $(p=0.602)$ and width of the distal radius $4 \mathrm{~cm}$ proximal to the radial styloid process tip $(p=0.335)$. No significant difference was observed between the volunteers who were righthanded and those who were left-handed in terms of the arc radii
Table I. Agreement between the measurements made by the principal investigator and radiologist.

\begin{tabular}{lcc}
\hline Parameter & ICC & $\mathbf{9 5 \%} \mathbf{~ C I}$ \\
\hline Arc radii (radial aspect) & 0.978 & $0.936-0.992$ \\
Arc radii (ulnar aspect) & 0.926 & $0.790-0.974$ \\
Curvature of the PQ line & 0.755 & $0.296-0.914$ \\
Greatest width of the distal radius & 0.960 & $0.884-0.986$ \\
Width of the distal radius 4 cm proximal & 0.935 & $0.543-0.982$ \\
to the radial styloid process tip & & \\
\hline
\end{tabular}

$\mathrm{Cl}$ : confidence interval; ICC: interclass correlation coefficient; $\mathrm{PQ}$ : pronator quadratus

on the radial aspect $(p=0.525)$, arc radii on the ulnar aspect $(p=0.525)$, curvature of the PQ line $(p=0.751)$, greatest width of the distal radius $(p=0.427)$ and width of the distal radius $4 \mathrm{~cm}$ proximal to the radial styloid process tip $(p=0.874)$.

\section{DISCUSSION}

While the morphology of the distal radius has been extensively studied, there is a lack of published studies detailing the arcs of the distal radius. Knowledge on the arcs of the distal radius is crucial in designing implants that can assist in achieving anatomical, or near anatomical, reduction of distal radius curvatures. To the best of our knowledge, the present study is the first study to utilise 3D-reconstructed CT images to examine morphological curvatures of the distal radii of healthy volunteers.

In the present study, a major concern was that healthy volunteers had to be subjected to radiation from CT. Interestingly, a recent study by Biswas et al revealed that, while CT of the axial and appendicular skeleton was associated with substantially elevated radiation exposure, the effective radiation dose declined substantially for CT done on anatomic structures that were further away from the torso. ${ }^{(14)}$ In that study, patients who underwent wrist CT were found to be exposed to only 0.38 times the radiation dose of conventional chest radiography. ${ }^{(14)}$ In other words, it is safer to undergo a wrist $\mathrm{CT}$ than conventional chest radiography, provided that necessary protective measures are taken.

In a recent study, Pichler et al reported that there was a statistically significant difference in the arc radii of the distal radius on the radial and ulnar sides in $55 \%$ of their cadaveric study population. ${ }^{(11)}$ The authors also highlighted that in $63 \%$ of the radii, the arc flattens towards the radial side. This interesting finding was also observed in a study by Gasse et al. ${ }^{(12)}$ In that study, the authors measured the volar metaphyseal-epiphyseal angles of the lateral and intermediate columns on sagittal CT images of the distal radius and found a statistically significant difference between the two slopes. Similarly, a statistically significant difference between the arc radii of the distal radius on the radial and ulnar aspects was found in the present study. If a different arc angle was defined as a difference of $5^{\circ}$ or more, $56.25 \%$ of the radii in the present study had a different arc angle. In addition, a deeper curve on the ulnar aspect was found in as many as $93.75 \%$ of the radii studied. In other words, the volar surface was observed to flatten toward the radial aspect in a majority of the cases. 
Table II. Comparison of the results of the present study and those of the study by Pichler et al.

\begin{tabular}{|c|c|c|}
\hline Parameter & Present study & Pichler et al, 2008(11) \\
\hline Measurement tool & $\begin{array}{l}\text { Computer software using } \\
\text { 3D-reconstructed CT images }\end{array}$ & $\begin{array}{l}\text { Profiles from cadavers were traced onto paper and measured } \\
\text { using curved templates with different radii }(1-6 \mathrm{~cm})\end{array}$ \\
\hline Arc radii (ulnar aspect) & $25.27^{\circ}\left(5.80^{\circ}\right)^{\dagger}$ & $2.3 \pm 1$ (range $1-6) \mathrm{cm}^{*}$ \\
\hline Greatest width of the distal radius* & $2.80 \pm 0.27 \mathrm{~cm}$ & $34.9 \pm 3.1$ (range $28-41) \mathrm{mm}$ \\
\hline $\begin{array}{l}\text { Width of the distal radius } 4 \mathrm{~cm} \text { proximal } \\
\text { to the radial styloid process tip* }\end{array}$ & $1.60 \pm 0.22 \mathrm{~cm}$ & $16.5 \pm 2.5$ (range $11-29) \mathrm{mm}$ \\
\hline Curvature of the $P Q$ line* & $40.52^{\circ} \pm 2.48^{\circ}$ & Not studied \\
\hline Differences between genders & $\begin{array}{l}\text { No significant differences } \\
\text { observed }\end{array}$ & $\begin{array}{l}\text { Men were found to have a wider distal radius than women, } \\
\text { but this difference was not statistically significant }\end{array}$ \\
\hline
\end{tabular}

*Data presented as mean \pm standard deviation. ${ }^{D}$ Data presented as median (interquartile range). 3D: three-dimensional; CT: computed tomography; PQ: pronator quadratus

Unfortunately, the present study was unable to determine a significant difference in the mean arc radii on the radial and ulnar aspects, between the Malaysian Malay and Western populations. This is because different research methodologies and measurement units were used. Pichler et $\mathrm{al}^{(11)}$ used circular profile gauges of different radii, while Gasse et al ${ }^{(12)}$ used the angle point at the site of maximal curvature after the tangent to the metaphysis and the epiphysis was drawn. In these two studies, the authors acknowledged that their measurements were just approximations, as they were measuring curves and not precise angles. A wellplanned multicentre trial is needed in order for valid comparisons of arc measurements to be made among different populations.

We did not find any significant gender distinction for all the measurements made in the present study (Table II). This is similar to the findings of Pichler et al's study, ${ }^{(11)}$ which also showed no gender distinction for the distal radius measurements, apart from measurements of the greatest width of the distal radius. The male subjects in Pichler et al's study, ${ }^{(11)}$ were observed to have a wider distal radius than the female subjects, but this difference was not found to be statistically significant. The collective results of both Pichler et al's study and the present study suggest that genderspecific implant designs are not needed.

Based on the data obtained in the present study, the design of volar fixed-angle plates can be optimised for use in the Malaysian Malay population. A distally convex curve to enable snug placement of the plate along the PQ line and separate sagittal bends for the radial and ulnar arcs may enhance the volar fixedangle plate's ability to achieve anatomical reduction in distal radius fractures, as such changes in design would reduce the likelihood of suboptimal or incorrect plate positioning.

To date, no single fixed-angle volar plate with different arcs to accommodate different arc radii for the radial and ulnar aspects has been marketed. The only implant that may address these curves adequately is the fragment-specific plating system, which was introduced in the 1990s. The use of these implants was supported by Rikli and Regazzoni ${ }^{(15)}$ when they introduced the concept that the distal radius and ulna form a three-column biomechanical construction that is made up of the radial, intermediate and ulnar columns. When the fixed-angle volar locking plates that are currently used are bent or recontoured, there is a risk that the plate holes will be distorted and the screw

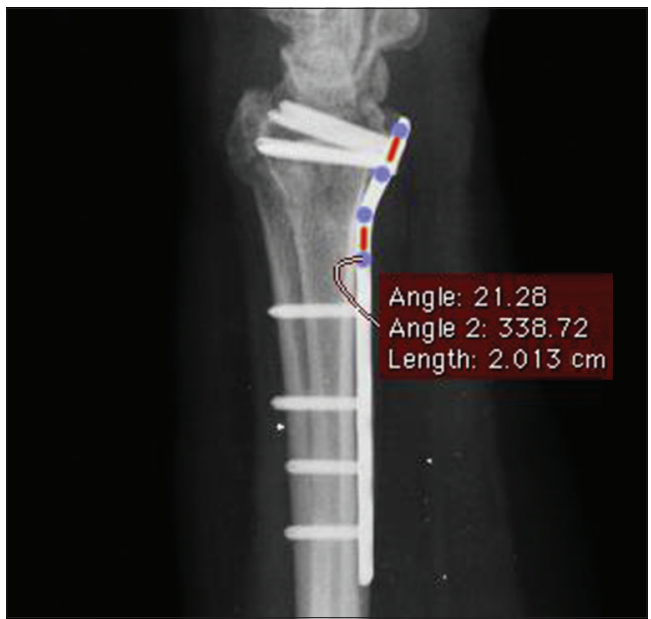

Fig. 6 Radiograph shows curve measurement for the Synthese ${ }^{\circ} \mathrm{T}$-Buttress locking compression volar plate, using the method in the present study.

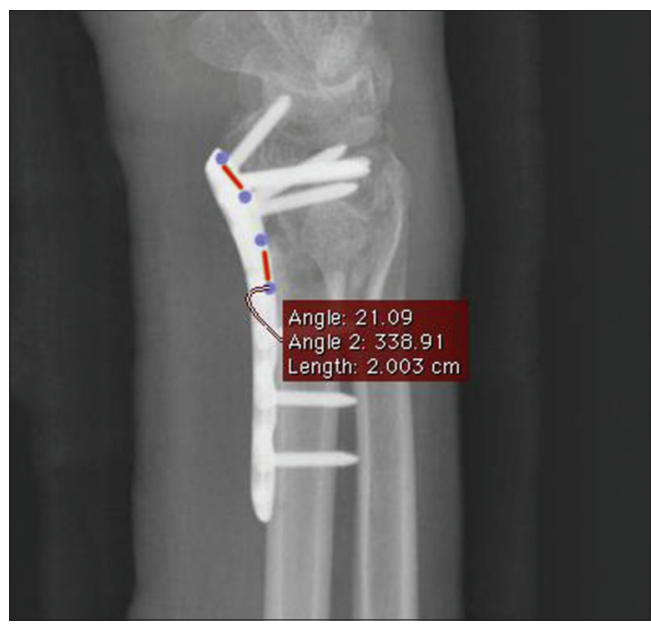

Fig. 7 Radiograph shows curve measurement for the Synthese ${ }^{\star}$ variableangle-locking, compression, two-column distal radius volar plate, using the method in the present study.

head prevented from being engaged. ${ }^{(16)}$ This is especially a problem when these implants, which are essentially designed for Caucasian wrists, are used on Asian wrists that are smaller and more mobile. ${ }^{(17)}$

When the measurement method used in the present study was used to measure the inner curves of two different fixed-angle volar plates, both readings were approximately $21^{\circ}$ (Figs. $6 \& 7$ ). This is 
approximately $4^{\circ}$ greater than the median arc radii on the radial aspect and $4^{\circ}$ less than the median arc radii on the ulnar aspect. While the significance of what appears to be a small discrepancy is still unclear, the current goal of osteosynthesis for distal radius fractures is to achieve anatomical reduction, particularly in the intermediate column.

The main limitation of the present study is its small sample size. Although the final sample size was extrapolated from statistical calculations, the underlying constraints were the costs (i.e. CT and radiographer expertise) and the risk of radiation exposure to the volunteers. In addition, convenience sampling was used; that is, all patients who fulfilled the inclusion criteria were recruited for this study. The present study also failed to compare the arc radii of Malaysian Malays with that of the Western population due to the different research methodologies and measurement units used. In addition, the heights and weights of the volunteers were not measured in the present study. These two parameters could have provided information regarding body size, which may have been associated with distal radius measurements.

There is still no definitive answer on the best treatment for displaced intra-articular distal radius fractures. Despite the lack of evidence-based medicine to support the use of fixed-angle volar plates in the management of these fractures, it is still the most popular and extensively used treatment. The treatment aim continues to be the achievement of anatomic alignment at the articular surface, with the hope of preventing radiographic changes. Nevertheless, the results of the present study can raise awareness of the changes that are required to optimise the design of this implant for the Malaysian Malay population.

\section{ACKNOWLEDGEMENTS}

We would like to express our gratitude to Dr Nor Azam Mahmad, Radiologist, SALAM Senawang Specialist Hospital, Negeri Sembilan, Malaysia, and Mr Mohd Arif Mustafa, Radiographer,
Department of Radiology, Universiti Sains Malaysia, Kelantan, Malaysia, for their contributions to this study.

\section{REFERENCES}

1. Drobetz $\mathrm{H}$, Bryant $A L$, Pokorny $T$, et al. Volar fixed-angle plating of distal radius extension fractures: influence of plate position on secondary loss of reduction--a biomechanic study in a cadaveric model. J Hand Surg Am 2006; 31:615-22.

2. Orbay JL, Touhami A. Current concepts in volar fixed-angle fixation of unstable distal radius fractures. Clin Orthop Relat Res 2006; 445:58-67.

3. Rozental TD, Blazar PE. Functional outcome and complications after volar plating for dorsally displaced, unstable fractures of the distal radius. J Hand Surg Am 2006; 31:359-65.

4. Rampoldi M, Marsico S. Complications of volar plating of distal radius fractures. Acta Orthop Belg 2007; 73:714-9.

5. Soong M, van Leerdam R, Guitton TG, et al. Fracture of the distal radius: risk factors for complications after locked volar plate fixation. J Hand Surg Am 2011; 36:3-9.

6. Simic PM, Weiland AJ. Fractures of the distal aspect of the radius: changes in treatment over the past two decades. Instr Course Lect 2003; 52:185-95.

7. Nana AD, Joshi A, Lichtman DM. Plating of the distal radius. J Am Acad Orthop Surg 2005; 13:159-71.

8. Cross AW, Schmidt CC. Flexor tendon injuries following locked volar plating of distal radius fractures. J Hand Surg Am 2008; 33:164-7.

9. Berglund LM, Messer TM. Complications of volar plate fixation for managing distal radius fractures. J Am Acad Orthop Surg 2009; 17:369-77.

10. Rhee PC, Dennison DG, Kakar S. Avoiding and treating perioperative complications of distal radius fractures. Hand Clin 2012; 28:185-98.

11. Pichler W, Clement H, Hausleitner L, et al. Various circular arc radii of the distal volar radius and the implications on volar plate osteosynthesis. Orthopedics 2008; 31

12. Gasse N, Lepage D, Pem R, et al. Anatomical and radiological study applied to distal radius surgery. Surg Radiol Anat 2011; 33:485-90.

13. Nelson DL, Orbay J, Bindra R. Anatomy of the Volar Distal Radius. eRADIUS - International Distal Radius Fracture Study Group. 2008.

14. Biswas D, Bible JE, Bohan M, et al. Radiation exposure from musculoskeletal computerized tomographic scans. J Bone Joint Surg Am 2009; 91:1882-9.

15. Rikli DA, Regazzoni P. Fractures of the distal end of the radius treated by internal fixation and early function. A preliminary report of 20 cases. J Bone Joint Surg Br 1996; 78:588-92.

16. Freeland AE, Luber KT. Biomechanics and biology of plate fixation of distal radius fractures. Hand Clin 2005; 21:329-39.

17. Sebastin SJ, Chung KC. An Asian perspective on the management of distal radius fractures. Hand Clin 2012; 28:151-6. 